where $\nu_{k}$ and $t_{k}$ are defined by a broken line approximation of $s$. If the initial conditions of (8) defining $s$ are changed to $\dot{s}(0)=s_{0}$ and $s(0)=s_{0}$, then (9) takes the following form:

$$
x=-\frac{\dot{s}_{0}}{\omega} \sin \omega t-s_{0} \cos \omega t+s-\omega \int_{0}^{t} s \sin \omega(t-\tau) d \tau,
$$

which again can be expressed as a sum of sinusoids of the same frequency.

On the basis of limited experience, the following suggestions for computation seem good. If the curve is sufficiently smooth, then the term containing $\mu_{1}$ will make a sizeable contribution; consequently the first time interval should be as small as convenient. It seems best to take $A(0)=a(0)$. The vector polygon will obviously be simplest if $t_{i}$ is selected so that as many values as possible of $\omega t_{i}$ are multiples of $\pi$.

If we had assumed $A(0) \neq 0$, then it would follow that

$$
\omega D=A-A(0) \cos \omega t-\frac{1}{\omega} \sum_{i=0}^{j-1}\left(\mu_{i+1}-\mu_{i}\right) \sin \omega\left(t-t_{i}\right) .
$$

If we let $\dot{s}_{k}=\dot{s}\left(t_{k}\right)$ then it is clear that $\nu_{k+1} \approx \xi_{k+1}$. Substituting in (9) we have

$$
x \approx \frac{1}{\omega} \sum_{k=0}^{j-1}\left(\xi_{k+1}-s_{k}\right) \sin \omega\left(t-t_{k}\right) .
$$

In calculating the maximum displacement, (11) would be more convenient than (9) since $s$ could be obtained by a single integration of $a(t)$.

\title{
A REMARK ON THE RECTIFICATION OF THE JOUKOWSKI PROFILE*
}

\author{
BY CHARLES SALTZER (Brown University)
}

The Joukowski profile is usually defined as the image under the Joukowski transformation,

$$
\zeta=z+c^{2} / z
$$

of a circle passing through the point $(-c, 0)$ whose center lies in the first quadrant, and whose radius is $c(1+\epsilon)$ where $c$, and $\epsilon>0$. Although this representation gives the complex potential of the incompressible flow about a Joukowski profile very readily, the representation of this profile as the inverse of a parabola ${ }^{1}$ has the advantage, as will be shown below, of introducing a parameter with direct geometrical meaning which permits the immediate rectification of the Joukowski profile in closed form.

In the $z_{1}$-plane consider the parabola

$$
y_{1}=\frac{1}{2} x_{1}^{2}
$$

It is interesting to note that the sum in (11) is the so-called left Cauchy-Stieltjes sum corresponding to $D$.

* Received Aug. 17, 1945.

'In this way the profile later called "Joukowski profile" was introduced by Chaplygin. See Chapylgin's Collected Papers, Leningrad 1933, vol. 2, pp. 144-178, in particular $\$ 6$. 
and the point $-(a+b i)$ as a center of inversion. The coefficient of $x_{1}^{2}$ may be taken as $1 / 2$ since it enters only as a scale factor. Only the case $a>0, b>-1 / 2$ will be treated here. ${ }^{2}$ The transformation

$$
\zeta_{1}=\left(z_{1}+a+i b\right)^{-1}
$$

maps the exterior of the parabola in the $z_{1}$-plane on the exterior of a Joukowski profile in the $\zeta_{1}$-plane. For the proper choice of parameters the profiles in the $\zeta$ and $\zeta_{1}$-planes can be mapped on each other by a linear transformation and a reflection.

Letting $d s=\left|d \zeta_{1}\right|$ and $d s_{1}=\left|d z_{1}\right|$ we have for the element of arc length on the Joukowski profile by (2) and (3),

$$
d s=\left|z_{1}+a+i b\right|^{-2}\left|d z_{1}\right|=\frac{4\left(1+x_{1}^{2}\right)^{1 / 2}}{4\left(x_{1}+a\right)^{2}+\left(x_{1}^{2}+2 b\right)^{2}} d x .
$$

This expression can be simplified by separation into partial fractions. The roots of the denominator can be obtained by equating the latter to zero, transposing one term, extracting the square roots of both sides, and solving the two resulting quadratic equations. For the non-symmetrical case $(a>0)$ this enables us to write

$$
s\left(x_{1}\right)=A\left[I\left(\frac{\alpha}{\beta}-\beta,-\beta, d, x_{1}\right)-I\left(\frac{\alpha}{\beta}+\beta, \beta, f, x_{1}\right)\right],
$$

where

$$
I\left(m, p, q, x_{1}\right)=\int_{0}^{x_{1}} \frac{(x+m)\left(1+x^{2}\right)^{1 / 2}}{x^{2}+p x+q} d x
$$

and

$$
\begin{aligned}
& \alpha=\frac{\sqrt{2}}{g}\left[1+\left(1+4 a^{2} g^{4}\right)^{1 / 2}\right]^{1 / 2}, \quad \beta=\frac{4 a}{\alpha}, \quad g=(1+2 b)^{-1 / 2}, \\
& A=-8 \beta\left[\beta^{4}+4 \beta^{2}+\alpha^{2}\left(\beta^{2}+4\right)\right]^{-1}, d=\frac{1}{4}\left[\beta^{2}+(\alpha+2)^{2}\right], f=\frac{1}{4}\left[\beta^{2}+(\alpha-2)^{2}\right] .
\end{aligned}
$$

The integrand of (6) can be rationalized by the substitution

$$
x=\left(1-u^{2}\right) / 2 u
$$

which gives

$$
I\left(m, p, q, x_{1}\right)=-\frac{1}{2} \int_{1}^{r-x_{1}} \frac{\left(1+2 m u-u^{2}\right)\left(1+u^{2}\right)^{2}}{u^{2}\left[\left(u^{2}-p u-1\right)^{2}+\left(4 q-p^{2}\right) u^{2}\right]} d u,
$$

where $r=\sqrt{1+x_{1}}$. The factors of the denominator of this integrand can be found in the same way as the factors of the denominator of (4) were found, and the integration can be carried out directly after expanding (9) in partial fractions with linear and quadratic denominators. It may be remarked that the $I$ 's taken individually may not converge over the entire range of $u$.

The case $a=0$ gives a symmetrical Joukowski profile for which the rectification can be carried out in a simpler way. Here equation (4) becomes

2 This configuration represents the most frequently used Joukowski profiles to within a scale factor and a reflection. The case $a<0$, by reason of symmetry, can be regarded as a reflection of the case $a>0$, and the case $b \leqq-1 / 2$ can be treated in a way similar to the treatment of the case $b>-1 / 2$. 


$$
d s=4\left(1+x_{1}^{2}\right)^{1 / 2}\left[4 x_{1}^{2}+\left(x_{1}^{2}+2 b\right)^{2}\right]^{-1} d x_{1},
$$

and equation (5) is replaced by

$$
s\left(x_{1}\right)=\int_{0}^{x_{1}} g\left(1+x^{2}\right)^{1 / 2}\left[\left\{x^{2}+(1-1 / g)^{2}\right\}^{-1}-\left\{x^{2}+(1+1 / g)^{2}\right\}^{-1}\right] d x .
$$

Setting

$$
x=t\left(1-t^{2}\right)^{-1 / 2}
$$

we get, after simplifying and expanding in fractions with quadratic denominators,

$$
s\left(x_{1}\right)=g \int_{0}^{x_{1}}\left[\left(\sigma_{1}^{2}-t^{2}\right)^{-1}-\left(\sigma_{2}^{2}-t^{2}\right)^{-1}\right] d t,
$$

where

Therefore

$$
\sigma_{1}^{2}=1+g^{2}(1+2 g)^{-1}, \quad \sigma_{2}^{2}=1+g^{2}(1-2 g)^{-1} \text {. }
$$

$$
s\left(x_{1}\right)=g\left[\sigma_{1}^{-1} \tanh ^{-1} \cdot\left\{x_{1} \sigma_{1}^{-1}\left(1+x_{1}^{2}\right)^{-1 / 2}\right\}-\sigma_{2}^{-1} \tanh ^{-1}\left\{x_{1} \sigma_{2}^{-1}\left(1+x_{1}^{2}\right)^{-1 / 2}\right\}\right] .
$$

If we denote the slope of the parabola at the point $\left(x_{1}, x_{1}^{2} / 2\right)$ by $\tan \gamma$ and note that $x_{1}=\tan \gamma$, we can write $(15)$ as

$$
s(\gamma)=g\left[\sigma_{1}^{-1} \tanh ^{-1}\left(\sigma_{1}^{-1} \sin \gamma\right)-\sigma_{2} \tanh ^{-1}\left(\sigma_{2}^{-1} \sin \gamma\right)\right],
$$

where $s$ is measured from the point furthest from the trailing edge.

In order to introduce the usual parameters $\epsilon$ and $c$ for the symmetrical case of the Joukowski profile ${ }^{3}$ consider the circle in the $z$-plane,

$$
z(\phi)=c\left[\epsilon+(1+\epsilon) e^{2 \phi}\right]
$$

which is the image of a symmetrical Joukowski profile in the $\zeta$-plane. The distance between the leading and trailing edges of the Joukowski profile in the $\zeta$-plane is (recalling Eq. (1))

$$
\zeta[z(0)]-\zeta[z(\pi)]=\frac{4 c(1+\epsilon)^{2}}{1+2 \epsilon} .
$$

From (3) the corresponding length in the $\zeta_{1}$-plane is seen to be $1 / b$ (i.e. the length in the $\zeta_{1}$-plane of the image of the upper half of the imaginary axis in the $z_{1}$-plane). If the profile in the $\zeta_{1}$-plane is identified with the profile in the $\zeta$-plane then

$$
b=\frac{1}{4}(1+2 \epsilon)(1+\epsilon)^{-2} c^{-1} .
$$

If the vertex of the parabola in the $x_{1}$-plane which corresponds to the given Joukowski profile in the $\zeta$-plane is at the origin, and if the $x_{1}$-axis coincides with the tangent to the parabola at this point, then it is readily seen by comparing the positions of the profiles in the $\zeta$-plane and the $\zeta_{1}$-plane that

$$
\zeta_{1}=-i(\zeta+2 c) \text {. }
$$

Since $a=0$, Eq. (3) can be written

$$
z_{1}=\frac{1}{\zeta_{1}}-i b
$$

${ }^{3}$ See, for instance, H. Glauert, The elements of aerofoil and air screw theory, The University Press, Cambridge, 1930, pp. 71-75. 
The successive substitution into Eq. (21) of Eqs. (20), (1), (17), and (19) gives after simplification

$$
z_{1}=x_{1}+i y_{1}=\frac{\epsilon}{2 c(1+\epsilon)^{2}} \tan \frac{1}{2} \phi+i \frac{1}{4 c(1+\epsilon)^{2}} \tan ^{2} \frac{1}{2} \phi .
$$

This is the parabola

$$
y_{1}=c \epsilon^{-2}(1+\epsilon)^{2} x_{1}^{2} .
$$

Since a change in the value of $c$, effects only a change of scale in the $\zeta$-plane, $c$ may be taken without loss of generality as

$$
c=\frac{1}{2} \epsilon^{2}(1+\epsilon)^{-2},
$$

and this parabola becomes the one considered in Eq. (2). Setting this value of $c$ in (19) yields

$$
b=\frac{1}{2}(1+2 \epsilon) \epsilon^{-2} .
$$

Hence, by Eqs. (7) and (14),

$$
\sigma_{1}^{2}=(1+2 \epsilon)^{2} /(1+\epsilon)(1+3 \epsilon), \quad \sigma_{2}^{2}=1 /\left(1-\epsilon^{2}\right), \quad g=\epsilon /(1+\epsilon) .
$$

Formulae (15) and (16) are valid for $\sigma_{1}>0, \sigma_{2}>0$, i.e., for $\epsilon<1$ and thus include those profiles whose thicknesses is less than about $4 / 5$ of their lengths.

It may also be noted that in terms of the variable $\gamma$, the slope, $\theta(\gamma)$, and the curvature, $d \theta(\gamma) / d s$, for the symmetrical profile may be written as

$$
\begin{aligned}
\theta(\gamma) & =\gamma-\arctan \left\{\frac{4 \tan \gamma\left(\tan ^{2} \gamma+(1+2 \epsilon) / \epsilon^{2}\right)}{4 \tan ^{2} \gamma-\left[\tan ^{2} \gamma+(1+2 \epsilon) / \epsilon^{2}\right]^{2}}\right\}, \\
\frac{d \theta(\gamma)}{d s} & =\frac{\sec \gamma}{8 c}\left[(1+3 \epsilon)(1-\epsilon) \epsilon^{-2} \cos ^{4} \gamma+6 \cos ^{2} \gamma-3 \epsilon^{2} /(1+\epsilon)^{2}\right] .
\end{aligned}
$$

CORRECTION AND SUPPLEMENT TO OUR PAPER

\section{THE CYLINDRICAL ANTENNA: CURRENT AND IMPEDANCE*}

\section{Quarterly of Applied Mathematics 3, 302-335 (1946)}

BY RONOLD KING AND DAVID MIDDLETON (Harvard University)

Equation (58) should be written as follows:

$$
\psi \equiv \bar{\Psi}= \begin{cases}\left|\Psi_{K_{1}}(0)\right|=\left|\psi_{1}(0)\right| / \sin \beta h ; & \beta h \leqq \pi / 2 \\ \left|\Psi_{K 1}(h-\lambda / 4)\right|=\left|\psi_{1}(h-\lambda / 4)\right| ; & \beta h \geqq \pi / 2 .\end{cases}
$$

Two lines before this equation $\left|\psi_{1}(0)\right| / \sin \beta h$ should be written instead of $\left|\psi_{1}(0)\right|$.

These changes involve no alternations in the figures. However, the function $|\psi(0)|$ plotted in Fig. 11 to the left of $\beta h=\pi / 2$ is not the parameter of expansion $\psi$ defined by (58) as modified above and as indicated in the caption. The parameter of expansion $\psi$ as defined in (58) is plotted in Fig. 11a where the part to the right of $\beta h=\pi / 2$ is the same as in Fig. 11, the part to the left of $\beta h=\pi / 2$ is obtained from the curves in Fig. 11 by dividing by $\sin \beta h$.

* Received Jan. 25, 1946. 